\title{
Proceeding
}

Supplementary Issue: Rio 2016 Olympic Games First Anniversary Special Edition. Olympic Studies Forum, 4-5 August 2017. Santa Úrsula University. Rio de Janeiro, Brazil

\section{The transform project and the geospatial reaching in Brazil: The legacy of the Olympic education in Rio 2016 Games}

\author{
CRISTIANO MEIGA BELEM11,2,3,4 , LEONARDO PEROVANO CAMARG01,4,5, OTÁVIO GUIMARÃES \\ TAVARES DA SILVA ${ }^{1,4,5}$, WILLIAN KÉVINY SOUZA BERTÉ1 \\ ${ }^{1}$ Federal University of Espírito Santo - UFES, Brazil \\ ${ }^{2}$ Departamento de Linguagens, Cultura e Educação - Centro de Educação - DELC/ CE, Brazil \\ 3Programa de Desenvolvimento Científico e Tecnológico Regional - DCR da Fundação de Amparo à \\ Pesquisa e Inovação do Espírito Santo - FAPES, Brazil \\ ${ }^{4}$ Centro de Estudos em Sociologia das Práticas Corporais e Estudos Olímpicos - Cespceo, Brazil \\ ${ }^{5}$ Centro de Educação Física e Desportos - CEFD, Brazil
}

\begin{abstract}
The Ministry of Education and the Organizing Committee of the Olympic Games Rio 2016 created the Transform Project, it provided training for teachers and students from public and private schools throughout Brazil, offering teaching material about the Olympic and Paralympic Movement, publicizing the education with Olympic values, the experimentation of new sports and engaging students in Rio 2016 Games. The Project was developed through the use of technologies, by internet, allowing the access to the material in every school in Brazil. A legacy to the Olympic Education left by Rio 2016, however, something similar but in a smaller dimension had already been done in Brazil in 1999 and 2000, with the Olympic Education Manual in School, it was available in two books in internet, one for teacher and another one for the students and it was distributed through a homepage. Today it is possible to identify the places where downloads were done, in other words, geolocalize the material downloaded, estimating its reaching and relation with the geographic space. From the information obtained of the materials downloaded in its four dimensions - teacher, pedagogical coordinator, tutor and digital content - geospatial analyzes of the schools which had access to the material were done and their relation with the official education data, the geographic census and

Corresponding author. Federal University of Espirito Santo - UFES, Brasil.

E-mail: cmbelem@bol.com.br

Supplementary Issue: Rio 2016 Olympic Games First Anniversary Special Edition. Olympic Studies Forum, 4-5 August 2017. Santa Úrsula University. Rio de Janeiro, Brazil.

JOURNAL OF HUMAN SPORT \& EXERCISE ISSN 1988-5202

(c) Faculty of Education. University of Alicante

doi:10.14198/jhse.2018.13.Proc1.12
\end{abstract}

S144 | 2018 |Proc1| VOLUME 13

C 2018 University of Alicante 
economic data. The analysis of the dimension where the Project reached in Brazil and its geospatial relation with the territory verified that it was present in every Brazilian state and in more than half of their municipalities. Key words: TRANSFORM PROJECT, OLYMPIC EDUCATION, GEOTECHNOLOGIES AND OLYMPISM, RIO 2016.

\section{Cite this article as:}

Meiga Belem, C., Perovano Camargo, L., Guimarães Tavares Da Silva, O. \& Souza Berté, W.K. (2018). The transform project and the geospatial reaching in Brazil: The legacy of the Olympic education in Rio 2016 Games. Journal of Human Sport and Exercise, 13(1proc), S144-S152. doi:https://doi.org/10.14198/jhse.2018.13.Proc1.12 


\section{BRASIL: 0 legado da educação Olímpica nos Jogos Rio 2016}

\section{RESUMO}

O Ministério da Educação e o Comitê Organizador dos Jogos Olímpicos Rio 2016 criaram o Projeto Transforma que proporcionou a capacitação para os professores e alunos das redes pública e particular em todo Brasil, oferecendo material didático sobre o Movimento Olímpico e Paralímpico, divulgando a educação dos valores olímpicos, a experimentação de novos esportes e o envolvimento dos escolares nos Jogos Rio 2016. O Projeto foi desenvolvido através do uso das tecnologias, via internet, possibilitando 0 acesso ao material a todas escolas do Brasil. Um legado para Educação Olímpica deixado pelos Jogos Rio 2016, entretanto, algo semelhante, numa dimensão muito menor, já havia sido realizado no Brasil nos anos 99/00, com o Manual Educação Olímpica na Escola, que disponibilizava através da internet dois cadernos, um para professor e outro para alunos e era distribuído através de uma homepage. Hoje podemos identificar os locais onde foram realizados os downloads, ou seja, geolocalizar o material "baixado", como isso, estimar seu alcance e relações com o espaço geográfico. A partir das informações obtidas dos downloads dos materiais do Projeto Transforma, em suas 4 dimensões - professor, coordenador pedagógico, tutor e conteúdo digital foram realizadas análises geoespaciais das escolas que tiveram acesso ao material e suas relações com dados oficiais da educação, do censo geográfico e dados econômicos. Nas análises da dimensão do alcance do Projeto Transforma no Brasil e suas relações geoespaciais com o território verificou-se que o projeto esteve presente em todos estados brasileiros e em mais da metade dos seus municípios. Palavras-chave: PROJETO TRANSFORMA, EDUCAÇÃO OLÍMPICA, GEOTECNOLOGIAS E OLIMPISMO, RIO 2016. 


\section{INTRODUÇÃO}

A avaliação do legado olímpico nos países-sede, após a realização dos Jogos, encontra-se como recomendação da Agenda Olímpica 2020 no terceiro $\left(3^{\circ}\right)$ parágrafo da quarta $\left(4^{\mathrm{a}}\right)$ recomendação: "garantir o monitoramento do legado após os Jogos Olímpicos com o suporte dos Comitês Olímpicos Nacionais (NOC) e outras organizações externas" (COI, 2014). Assim, os países-sede deveriam preocupar-se em avaliar cientificamente quais os impactos foram deixados nos países após a realização dos Jogos, como forma de justificar os investimentos feitos, além de fornecer formas de planejar melhores edições futuras.

Um dos elementos que compõem este legado olímpico são as políticas públicas de educação olímpica, que são uma forma de atingir positivamente os estudantes de países participantes. Determinados países têm um monopólio de práticas esportivas e transmissão no meio de comunicação, deixando os jovens aquém das práticas que compõem uma Olimpíada.

Outra questão nesta análise é a visão reducionista dos Jogos Olímpicos como tendo impacto apenas no curto período de competições, ignorando sobremaneira as simbologias, valores e possibilidades educacionais contidas neste megaevento.

Pierre de Coubertin - fundador do Comitê Olímpico Internacional (COI), do Movimento Olímpico e dos Jogos Olímpicos da era moderna - considerava o esporte como elemento fundamental na educação dos jovens, como qualquer outra disciplina escolar. Esse Movimento Olímpico carrega este conceito de educação desenvolvido por Coubertin, sendo inclusive não dependente da existência dos Jogos (Miragaya, 2009).

Pode-se conceituar como Educação Olímpica "as propostas de educação através do esporte tendo como referência o Movimento Olímpico, seus valores declarados, seu simbolismo, sua história, seus heróis e suas tradições" (Tavares, 2008).

Assim, programas de educação Olímpica educam os jovens em valores olímpicos, além de prepará-los a acompanhar o evento com maior bagagem cultural, deixando um legado através da relação das experiências vividas no sistema escolar e do acompanhamento dos ideais olímpicos difundidos nos meios midiáticos.

Aliar a educação olímpica, através de políticas públicas nos países-sede, ao sistema educacional formal é uma realidade antiga, desde Los Angeles 86 , ampliando seu alcance em Pequim 2008. No Brasil, o primeiro programa dessa natureza a ser desenvolvido foi o Educação Olímpica na Escola, criado pelo Professor Cristiano Belem, em 1998, com objetivos ligados às atitudes, valores e olimpismo. Os materiais desenvolvidos foram um manual de Educação Olímpica difundido pela internet, o Manual do Educador e um Caderno de Atividades em Educação Olímpica, para aplicação na Educação Física escolar em todo território brasileiro (Belem, 1999).

Nos Jogos Rio 2016 foi utilizado um Programa de Educação, denominado Projeto Transforma, que levou os Jogos Olímpicos e Paralímpicos Rio 2016 para dentro das escolas. O Projeto atuou em parceria com as escolas, criando oportunidades para estudantes de Ensino Fundamental e Médio vivenciarem os Valores Olímpicos e Paralímpicos, experimentarem novos esportes e engajarem-se nos Jogos. 0 Projeto Transforma teve a parceria do Ministério da Educação Brasileiro e do Comitê Organizador dos Jogos Olímpicos Rio 2016. O objetivo do Projeto foi levar capacitação em educação olímpica para os professores 
das redes pública e privada, levando diversificação, também, no oferecimento de modalidades esportivas nas escolas. ${ }^{1}$

A formação oferecida pelo Projeto Transforma foi toda online e gratuita, com uma diversidade de opções entre materiais didáticos, cursos de formação, capacitações esportivas, desafios escolares, simbologia e valores olímpicos e paralímpicos. O conteúdo disponibilizado foi organizado em 4 tipos de materiais, destinado ao professor de educação física escolar, ao coordenador pedagógico escolar, aos tutores de agentes jovens e um conteúdo com atividades, denominado conteúdo digital.

O mecanismo de distribuição do material foi realizado mediante login do interessado com a obrigatoriedade de informar a escola que se destinava o download do conteúdo do Projeto Transforma. Esse mecanismo de distribuição do download permitiu através de ferramentas da geoinformação uma análise mais abrangente da divulgação dos conteúdos do Projeto no Brasil, ou seja, analisar o impacto da divulgação e distribuição dos conteúdos do Projeto Transforma no território nacional.

Embora existam estudos sobre a importância e a necessidade do alcance das políticas públicas de educação olímpica em todo o território dos países-sede, como também alguns estudos que apontam a influência de forma qualitativa, há uma dificuldade na quantificação do alcance das mesmas.

Em um período onde há uma extrema racionalização econômica, estabelecer modelos de análise e oferecer dados para o poder público avaliar e tomar decisões com base em dados é uma justificativa importante para estes estudos.

Diante disso, a proposta deste estudo foi utilização de uma análise quantitativa a partir do uso das geotecnologias, utilizando-se dos dados de identificação dos usuários que realizaram download dos conteúdos na plataforma online do Projeto Transforma, a fim de compreender o impacto geoespacial deste programa no território brasileiro.

\section{MATERIAIS E METODOLOGIA}

A avaliação do alcance do Projeto Transforma se deu pela análise das inscrições online. O responsável por baixar os materiais precisava citar a qual escola do território nacional estava vinculado. Estes dados foram vinculados aos Microdados do Censo 2016 do Instituto Nacional de Estudos e Pesquisas Educacionais Anísio Teixeira (INEP), com dados das escolas, dos alunos e dos professores.

Com objetivo de observar a amplitude da distribuição dos conteúdos do Projeto Transforma, os dados foram pontuados no espaço geográfico através de ferramentas de Sistema de Informação Geográfica (SIG), na qual foram realizadas análises geoespaciais para geração de informações dos municípios, dos estados e das regiões.

O termo SIG, segundo Câmara, Druck, Carvalho e Monteiro (2002), é aplicado para sistemas que realizam 0 tratamento de dados geográficos e relacionam a geometria e 0 atributo dos dados que foram georreferenciados. Dessa forma, foram realizadas algumas operações como juntar, resumir e deletar

1Projeto treinará professor para diversificar esportes na escola. Disponível em portal.mec.gov.br/component/tags/tag/36392, acesso em 12/10/2017. 
campos, criando-se arquivos shapefile ${ }^{2}$ com informações dos dados relacionados a geometrias espacializadas numa projeção cartográfica. Com estes arquivos, foi possível desenvolver mapas temáticos para avaliar o impacto do Projeto Transforma. A avaliação se baseou em estudar 4 parâmetros, sendo eles: os documentos baixados para uso por professores, coordenadores e tutores, e os documentos de informações gerais - conteúdo digital.

\section{APRESENTAÇÃO DOS DADOS}

A figura 1 apresenta a distribuição geográfica nos estados brasileiros onde foram realizados downloads dos 4 conteúdos disponibilizados pelo Projeto Transforma. Os dados da origem dos downloads, contendo a informação sobre a qual escola destinaram-se os conteúdos, foram geoespacializados para serem estudados através de análises geoespaciais. A figura 1 mostra como se comportaram os downloads dos documentos específicos voltados aos professores de Educação Física, aos coordenadores, tutores e do conteúdo digital. Verificou-se que para todos os 4 modelos de conteúdos, o estado de Minas Gerais foi 0 que mais realizou downloads, seguido pelos estados de São Paulo e Rio de Janeiro. Os estados que realizaram menor quantidade de downloads foram os estados do Acre, Tocantins, Rondônia, Roraima, Amapá, Alagoas e Sergipe. Observando a figura 1, percebe-se que o projeto esteve mais presente na região geoeconômica centro-sul do Brasil, enquanto as regiões da Amazônia e do nordeste não foram assim tão influenciadas.
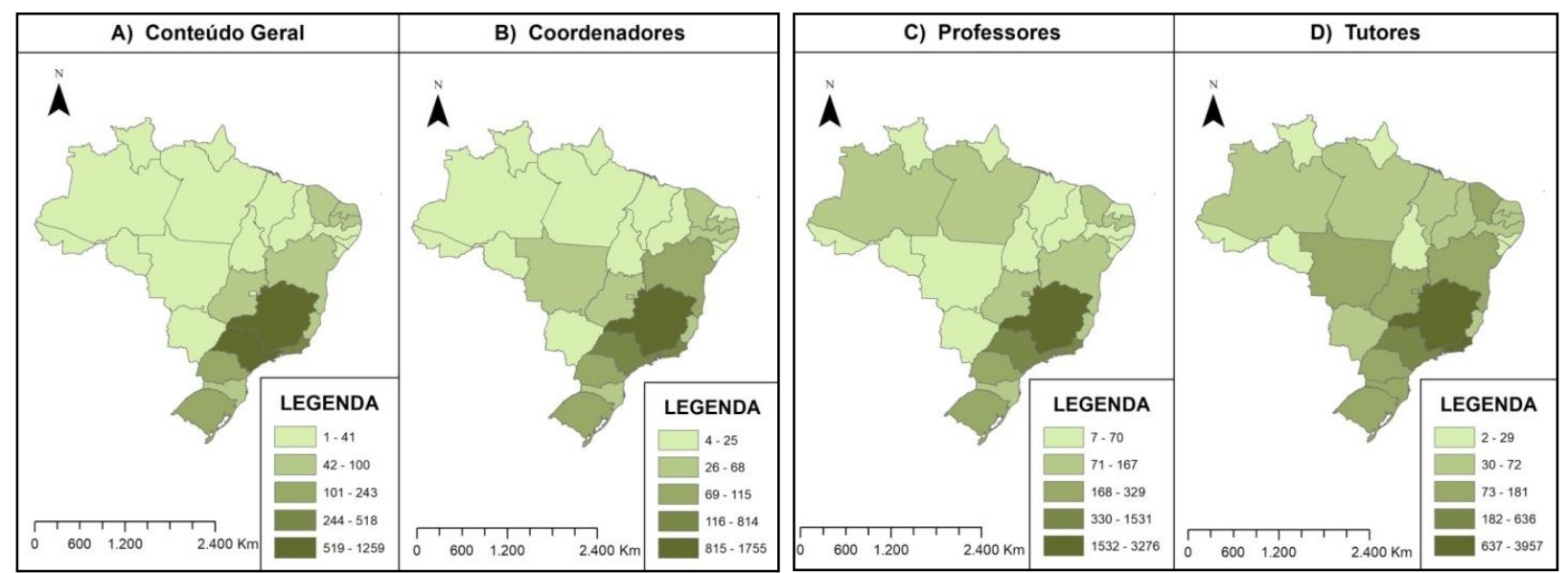

A: Representação gráfica da quantidade de downloads de conteúdo geral. B: Número de coordenadores atingidos pelo programa que baixaram materiais destinados a coordenadores.C: Mapa com a representação dos professores envolvidos no Transforma que fizeram download do material para professores. D: Quantidade de Tutores que baixaram arquivos para tutores.

Figura 1. Mapas de influência do Projeto Transforma.

A figura 2 apresenta a relação numérica entre 0 total de alunos e professores de Educação Física que possam ter tido contato com o material do Projeto Transforma, a partir dos dados de downloads realizados.

2 Um shapefile é um formato de armazenamento de dados de vetor da ESRI para armazenar a posição, forma e atributos de feições geográficas. A ESRI é uma empresa americana especializada na produção de soluções para a área de informações geográficas, sendo líder mundial em sistemas de informação geográfica. 


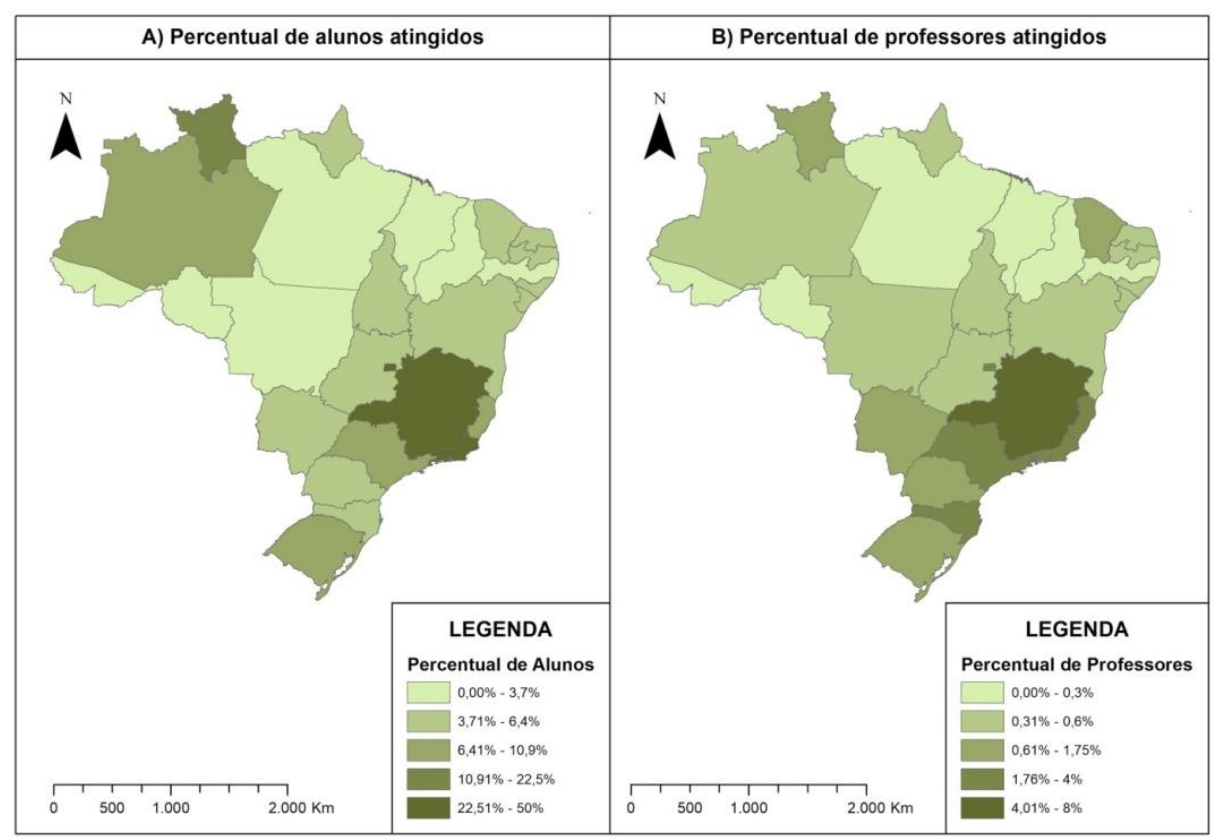

A: Apresenta o percentual de alunos alcançados. B: Apresenta o percentual de professores de Educação Física alcançado pelo projeto.

Figura 2. Percentual de alunos e professores atingidos pelo Transforma.

$\mathrm{Na}$ tabela abaixo, observa-se 0 alcance do Projeto Transforma nas capitais dos estados brasileiros. Podese perceber que as capitais do sudeste, com exceção de Vitória- ES, foram as mais influenciadas em números absolutos, além disso, Manaus e Brasília foram as cidades fora do Sudeste que mais foram impactadas. Aos dados foram acrescidos o quantitativo de alunos nas escolas das capitais brasileiras, onde foram realizados download do conteúdo de professores, cuja soma ultrapassa 2 milhões de alunos.

Tabela 1. Impacto do Projeto Transforma nas capitais brasileiras

\begin{tabular}{|l|l|l|l|l|l|l|l|l|}
\hline Município & UF & População & $\begin{array}{l}\text { PIB } \\
\text { (em mil reais) }\end{array}$ & Coordenador & Conteúdo & Tutor & Professor & Alunos* \\
\hline Aracaju & SE & 571.149 & 8.751 .494 & 2 & 2 & 7 & 3 & 4518 \\
\hline Belém & PA & 1.393 .399 & 17.987 .323 & 3 & 3 & 8 & 17 & 17066 \\
\hline Belo Horizonte & MG & 2.375 .151 & 51.661 .760 & 170 & 103 & 397 & 844 & 695920 \\
\hline Boa Vista & RR & 284.313 & 4.659 .977 & 5 & 10 & 15 & 26 & 20208 \\
\hline Brasília & DF & 2.570 .160 & 149.906 .319 & 59 & 32 & 96 & 217 & 274526 \\
\hline Campo Grande & MS & 786.797 & 13.875 .046 & 5 & 12 & 11 & 12 & 10549 \\
\hline Cuiabá & MT & 551.098 & 11.051 .628 & 5 & 4 & 13 & 9 & 7718 \\
\hline Curitiba & PR & 1.751 .907 & 53.106 .497 & 31 & 73 & 29 & 57 & 67686 \\
\hline Florianópolis & SC & 421.240 & 9.806 .534 & 11 & 12 & 11 & 25 & 19015 \\
\hline Fortaleza & CE & 2.452 .185 & 37.106 .309 & 10 & 22 & 28 & 34 & 30523 \\
\hline Goiânia & GO & 1.302 .001 & 24.445 .744 & 17 & 22 & 23 & 30 & 18543 \\
\hline João Pessoa & PB & 723.515 & 9.805 .587 & 7 & 17 & 23 & 28 & 20612 \\
\hline Macapá & AP & 398.204 & 5.215 .130 & 3 & 0 & 8 & 6 & 3975 \\
\hline
\end{tabular}




\begin{tabular}{|l|l|l|l|l|l|l|l|l|}
\hline Maceió & AL & 932.748 & 12.114 .090 & 8 & 10 & 7 & 9 & 8429 \\
\hline Manaus & AM & 1.802 .014 & 48.598 .153 & 6 & 29 & 25 & 80 & 91906 \\
\hline Natal & RN & 803.739 & 11.997 .401 & 4 & 20 & 11 & 17 & 15528 \\
\hline Palmas & TO & 228.332 & 3.927 .446 & 0 & 2 & 12 & 8 & 7.963 \\
\hline Porto Alegre & RS & 1.409 .351 & 43.038 .100 & 12 & 43 & 13 & 44 & 3878 \\
\hline Porto Velho & RO & 428.527 & 7.522 .929 & 5 & 3 & 1 & 5 & 3650 \\
\hline Recife & PE & 1.537 .704 & 30.032 .003 & 3 & 6 & 16 & 8 & 8465 \\
\hline Rio Branco & AC & 336.038 & 4.311 .124 & 2 & 4 & 2 & 4 & 3551 \\
\hline Rio de Janeiro & RJ & 6.320 .446 & 190.249 .043 & 331 & 163 & 2021 & 429 & 337231 \\
\hline Salvador & BA & 2.675 .656 & 36.744 .670 & 5 & 1 & 1 & 3 & 95.65 \\
\hline São Luís & MA & 1.014 .837 & 17.915 .048 & 10 & 9 & 5 & 9 & 7707 \\
\hline São Paulo & SP & 11.253 .503 & 443.600 .102 & 58 & 216 & 85 & 226 & 215887 \\
\hline Teresina & PI & 814.230 & 10.539 .378 & 4 & 5 & 7 & 10 & 10725 \\
\hline Vitória & ES & 327.801 & 24.969 .295 & 6 & 5 & 4 & 4 & 4940 \\
\hline
\end{tabular}

${ }^{*}$ Quantidade de alunos nas escolas com professores envolvidos no projeto - total de alunos 2.040.184

\section{DISCUSSÃO}

Com o uso das geotecnologias foi possível quantificar e verificar no espaço geográfico do território brasileiro a possível distribuição dos conteúdos do Projeto Transforma na população escolar. Com isso pode-se estimar 0 alcance do programa de educação olímpica nas escolas brasileiras.

Ferramentas que favoreçam a gestão dos recursos e a melhora das informações sobre as ações e programas públicos podem prover análises racionais de políticas públicas (Dye, 2005) e se fazem necessárias em uma sociedade que pede transparência e competência na gestão pública.

A proposta do estudo foi a apresentação da ferramenta de geotecnologia como um mecanismo de análise na distribuição de quantitativa do Projeto Transforma, cujo objetivo era difundir um programa de educação olímpica nas escolas brasileiras para os Jogos Olímpicos e Paralímpicos Rio 2016.

\section{CONCLUSÃO}

Conclui-se, através dos dados obtidos, que o Projeto Transforma atingiu todo o território brasileiro e uma parcela significativa da população escolar. Porém, há a necessidade do feedback das escolas ou uma análise mais profunda para a confirmação dessa permeabilidade do Projeto Transforma nas escolas e da difusão dos conteúdos, bem como dos valores dos jogos olímpicos e paralímpicos entre os escolares. Novas pesquisas baseadas em crowdsourcing podem ser um caminho para o aprimoramento das pesquisas que utilizem do geoferrenciamento como opção metodológica.

\section{REFERENCES}

Belem, C. M. (1999). Educação Olímpica na Escola. Adaptado de "Keep the Spirit Alive You and the Olympic Games" (I. M. Silvestre, Trad.). Poços de Caldas, MG. 
Câmara, G., Druck, S., Carvalho, M.S., \& Monteiro, A.V.M. (Eds.). (2004). Análise Espacial de Dados Geográficos. Brasília: EMBRAPA.

Comitê Olímpico Internacional. (2014). Olympic Agenda 2020: background and context. COI. Obtido em 12 de Outubro de 2017.

Dye, T.R. (2005). Models of politics: some help in thinking about public policy. In T.R. Dye. Understanding public policy. (11a. ed.) New Jersey: Prentice-Hall.

Miragaya, A. (2009). Educação Olímpica: o legado de Coubertein no Brasil. In A.R. Reppold Filho, L.M.M. Pinto, R.P. Rodrigues \& S. Engelman. (Orgs.). Olimpismo e Educação Olímpica no Brasil (pp.41-54). Porto Alegre: Editora da UFRGS.

Tavares, O. (2008). Educação Olímpica no Rio de Janeiro: notas iniciais para o desenvolvimento de um modelo. In R.P. Rodrigues, L.M.M. Pinto, R. Terra \& L.P. DaCosta (Orgs.). Legados de Megaeventos Esportivos (pp.343-356). Brasília: Ministério dos Esportes.

\section{(c) $(i)(9)$}

This title is licensed under a Creative Commons Attribution-NonCommercial-NoDerivs 3.0 Unported License. 